\title{
Substrate effects on the formation of flat Ag films on (110) surfaces of III-V compound semiconductors
}

\author{
K.-J. Chao \\ Department of Physics, University of Texas at Austin, Austin, Texas 78712 \\ Zhenyu Zhang \\ Solid State Division, Oak Ridge National Laboratory, Oak Ridge, Tennessee 37831 \\ and Department of Physics and Astronomy, University of Tennessee, Knoxville, Tennessee 37996 \\ Ph. Ebert \\ Department of Physics, University of Texas at Austin, Austin, Texas 78712 \\ and Institut für Festkörperforschung, Forschungszentrum Jülich, 52425 Jülich, Germany \\ C. K. Shih \\ Department of Physics, University of Texas at Austin, Austin, Texas 78712
}

(Received 29 March 1999)

\begin{abstract}
Ag films grown at $135 \mathrm{~K}$ on (110) surfaces of III-V compound semiconductors and annealed at room temperature are investigated by scanning tunneling microscopy and low-energy electron diffraction. Ag films on $\mathrm{Ga}-\mathrm{V}$ semiconductors are well ordered, atomically flat, and exhibit a specific critical thickness, which is a function of the substrate material. Films grown on In-V semiconductors are still rather flat, but significantly more disordered. The (111) oriented Ag films on III-arsenides and III-phosphides exhibit a clear twofold superstructure. Films on III-antimonides exhibit threefold low-energy electron diffraction images. The morphology of the Ag films can be explained on the basis of the electronic growth mechanism.
\end{abstract}

[S0163-1829(99)10831-2]

\section{INTRODUCTION}

The desire to achieve a precise control of each atomic layer during growth of thin films has initiated considerable research. On the one hand, much progress has been made for a variety of metal-metal and semiconductor-semiconductor systems, where the energetics of individual atoms and molecules were found to govern the overall growth mode. On the other hand, it is much more difficult to obtain atomically flat metal overlayers on semiconductors. Recently it has been shown that using a two-step growth process-depositing silver at low temperatures and annealing at room temperature-yields atomically flat silver films on GaAs (110) surfaces. ${ }^{1}$ In that case it turned out that the quantummechanical states of delocalized electrons dictate the macroscopic atomic arrangement. ${ }^{2}$ This so-called electronic growth mechanism is in agreement with the observed critical thickness of the silver film on GaAs(110) of $1.5 \mathrm{~nm}$ and the observation of quantum size effects in photoemission spectra. ${ }^{3}$ Furthermore the atomically flat silver film on $\operatorname{GaAs}(110)$ exhibits a quasiperiodic modulation of the (111) silver planes. Therefore it is highly desirable to determine if such atomically flat silver films occur on different substrate materials, too, and what structure these metal films have.

In this paper we report on an investigation of the substrate effects on the formation of flat $\mathrm{Ag}$ films on a variety of indium and gallium (110) surfaces containing III-V compound semiconductors. We demonstrate that flat Ag films can be obtained on several of the III-V semiconductor substrates by growing a Ag film at low temperatures and anneal- ing at room temperature. Each substrate material induces, however, a particular structure of the silver overlayer, a different critical thicknesses, and different degrees of disorder of the films. Ga-containing III-V semiconductors exhibit well ordered $\mathrm{Ag}$ overlayers, whereas $\mathrm{Ag}$ films on indium containing III-V semiconductors are rather disordered. The results can be explained by the different electronic properties of the substrate materials affecting the electronic growth mechanism.

\section{EXPERIMENT}

The different substrates were obtained by cleaving GaAs, $\mathrm{GaP}, \mathrm{GaSb}$, InAs, InSb, and InP single crystal bars along (110) planes in ultrahigh vacuum. On the cleaved surfaces Ag was deposited at $135 \mathrm{~K}$ with a deposition rate between 0.03 and $0.15 \mathrm{~nm} / \mathrm{min}$. The samples were then warmed up to room temperature and investigated by scanning tunneling microscopy (STM) and low-energy electron diffraction (LEED). The STM images were obtained at constant currents of typically $0.2 \mathrm{nA}$ and tunneling voltages of typically $0.5-2$ $\mathrm{V}$. The LEED images were acquired at energies ranging between 50 and $190 \mathrm{eV}$.

\section{RESULTS}

Figure 1 shows the morphology of the Ag layer on different Ga-V semiconductor substrates. On GaAs (110) surfaces the deposition of $\mathrm{Ag}$ at low temperatures leads to very flat films [Fig. 1(b1)]. Any film with a nominal thickness below $1.5 \mathrm{~nm}$ exhibits 1.5 -nm-deep holes where no $\mathrm{Ag}$ is found [see holes in Fig. 1(b1)]. On the bottom of these holes the 

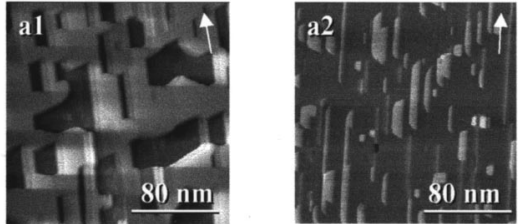

a3
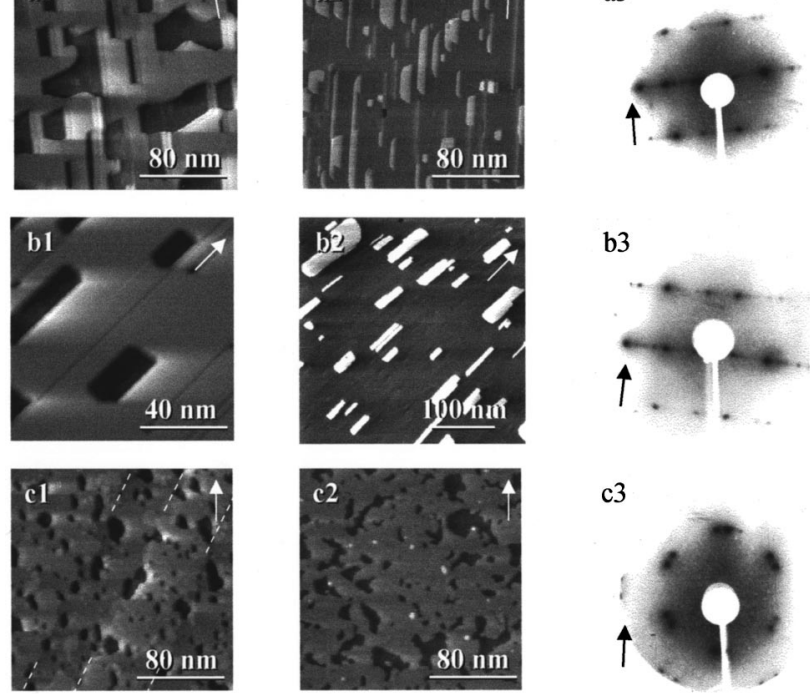

c3

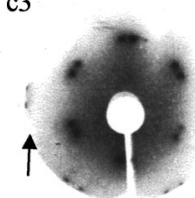

FIG. 1. Constant-current STM images (frames marked 1 and 2) and LEED images (frames 3) of Ag films grown on (a) $\mathrm{GaP}$, (b) GaAs, and (c) GaSb (110) surfaces at low temperatures. Frames marked by 1 show the surface structure for Ag films below the critical thickness, frames marked 2 show the morphology of $\mathrm{Ag}$ films above the critical thickness. The LEED images were obtained at energies of (a3) 106.5, (b3) 105, and (c3) $135.2 \mathrm{eV}$. The orientation of the STM images differ from each other and from that of the LEED images. The arrows in each frame indicate the $\langle 1 \overline{1} 0\rangle$ directions. The film thicknesses are (a1) 1.0, (a2) 2.0, (a3) 1.7, (b1) 1.5, (b2) 1.7, (b3) 1.7, (c1) 0.5, (c2) 1.0, and (c3) $1.7 \mathrm{~nm}$.

clean GaAs (110) structure can be recognized. From this we conclude that the $\mathrm{Ag}$ films on $\mathrm{GaAs}(110)$ have a critical thickness of $1.5 \mathrm{~nm}$. Here the term critical thickness has an opposite meaning for the same term in the context of pseudomorphic growth, where flat films can only be grown below a certain critical thickness. In our case films with a thickness below the critical or threshold thickness yield an atomically flat film or islands (depending on the nominal coverage) with exactly the critical thickness [see Fig. 1(b1)]. Films grown with larger thicknesses [Fig. 1(b2)] exhibit one atomic layer high islands of $\mathrm{Ag}$ on the 1.5-nm-thick $\mathrm{Ag}$ film. These films are still atomically flat. The Ag film has a (111) orientation according to the LEED image in Fig. 1(b3) and highresolution STM images. ${ }^{1}$ The (110) planes exhibit, however, a height modulation with quasiperiodic sequences that give rise to the additional spots on the LEED image. This effect induces also the apparent twofold morphology of the holes and islands in and on the Ag film. The Ag film is well ordered as the LEED spots are sharp within the limited resolution of the LEED system.

On $\mathrm{GaP}(110)$ surfaces the Ag films exhibit many similarities [see Figs. 1(a1)-1(a3)]. In particular, atomically flat $\mathrm{Ag}$ films are observed. The films exhibit again a critical thickness and are well ordered since sharp LEED spots are found. However, some differences occur. First the critical thickness of the $\mathrm{Ag}$ film is $1.7 \mathrm{~nm}$, i.e., one atomic layer more than on GaAs. Figure 1(a1) shows that films below the critical thickness exhibit the 1.7-nm-deep holes exposing the bare $\mathrm{GaP}(110)$ surface. The Ag film on GaP exhibits a pronounced twofold morphology even if the Ag film has, as in a

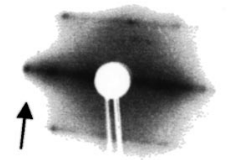

b

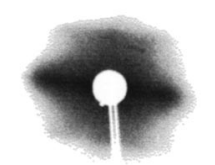

c

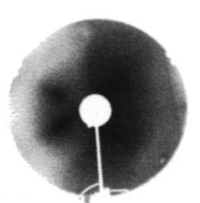

FIG. 2. LEED images of Ag films grown at low temperatures on (a) InP, (b) InAs, and (c) InSb (110) surfaces. The thicknesses of the Ag films are (a) 1.2, (b) 2.1, and (c) $3.5 \mathrm{~nm}$. The arrow indicates the $\langle 1 \overline{1} 0\rangle$ direction in real space.

the case of GaAs, a (111) orientation [see the LEED image in Fig. 1(a3)]. The additional spots in between the major ones of the (111)-oriented Ag film are again due to the superstructure of the Ag film. However, a close look shows that the Ag film does not exhibit a well-defined quasiperiodic order. Films grown above the critical thickness also exhibit one atomic layer high islands of $\mathrm{Ag}$ on the 1.7-nm-thick Ag film [Fig. 1(a2)]. The islands have a high anisotropy and are elongated along the $[1 \overline{1} 0]$ direction. The anisotropy of the surface is again reflected in the LEED image showing lines.

On GaSb (110) surfaces the structure of the Ag film is considerably different. First we observed two different domains leading to a slight spatial difference in the contrast. In Fig. 1(c1) three domain boundaries are marked by dashed line. The presence of domains is also confirmed by the LEED images, which exhibit a clear threefold surface of two domains rotated by about $10^{\circ}$. No other spots are observed and thus no superstructure exists. The Ag films on $\mathrm{GaSb}$ have always a (111) orientation in both domains and the LEED images indicate a high order. Films with nominal thickness of less than $0.6 \mathrm{~nm}$ exhibit $0.6-\mathrm{nm}$-deep holes, which exhibit no particular twofold shape. Thus the critical thickness is only about $0.6 \mathrm{~nm}$ compared to $1.5 \mathrm{~nm}$ for GaAs and $1.7 \mathrm{~nm}$ for GaP. No anisotropy of the film is found. Films grown with thicknesses above $0.6 \mathrm{~nm}$ exhibit again one atomic layer high steps.

The structure of Ag films grown at low temperatures on (110) surfaces of indium- $\mathrm{V}$ semiconductors exhibits a significantly different behavior. Figure 2 shows LEED images of Ag films grown on (a) InP, (b) InAs, and (c) InSb (110) surfaces using the same procedure as for the films shown in Fig. 1. For all three In-V semiconductor substrates the LEED images of the Ag films indicate a considerably increased degree of disorder of the films compared to those on the respective Ga containing III-V semiconductors. In case of InAs and InP a twofold symmetry is observed similar to that found for Ag films on GaAs and $\mathrm{GaP}$ (110) surfaces. For InSb a weak threefold structure is observed in LEED images comparable to the clear threefold structure on GaSb. The highest degree of order, reflected in the sharpest LEED spots, is observed for Ag films on $\operatorname{InP}(110)$. Figure 3 shows a STM image of the structure of such a $\mathrm{Ag}$ film on $\operatorname{InP}(110)$. Compared to the STM images in Fig. 1 it is obvious that the film has a considerably increased disorder. We note, however, that the Ag film is still very flat. In particular, the Ag film grown at low temperatures and annealed at room temperature on $\operatorname{InP}(110)$ is significantly smoother than $\mathrm{Ag}$ films grown at room temperature on (110) surfaces of III-V compound semiconductor. ${ }^{4}$ 


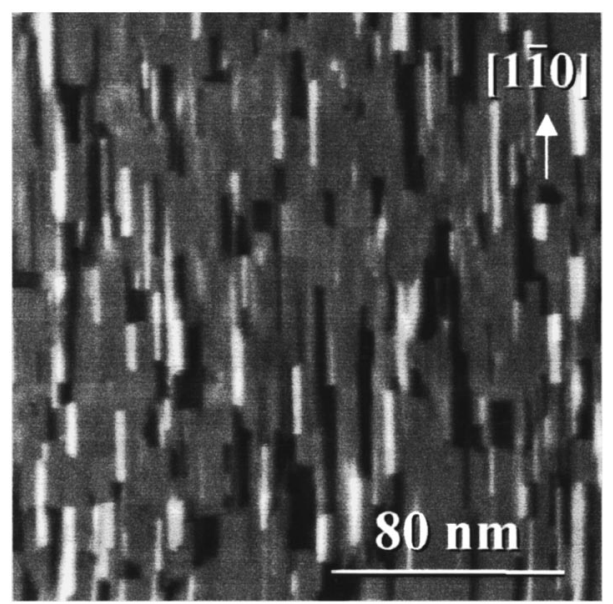

FIG. 3. Constant-current STM image of a 2.1-nm-thick Ag film on an $\operatorname{InP}(110)$ surface grown at low temperature and annealed at room temperature.

\section{DISCUSSION}

The experimental results are summarized in Table I. Table I highlights the following trends on which our discussion will focus. First, Ag films on Ga containing compound semiconductors are significantly better ordered (and exhibit a critical thickness) than those on the respective In-containing semiconductors. The disorder of the $\mathrm{Ag}$ films on In-V semiconductors indicates the absence of a critical thickness of the $\mathrm{Ag}$ film. Second, the critical thickness on Ga-V semiconductor decreases with increasing lattice constant. Finally the IIIphosphides and III-arsenides substrates lead to a twofold superstructure, whereas the III-antimonides exhibit a threefold LEED pattern.

The formation of flat $\mathrm{Ag}$ films on $\mathrm{GaAs}(110)$ surfaces using low-temperature growth and annealing at room temperature has been explained on the basis of an electronic growth mechanism. ${ }^{2}$ This model predicts that the overall morphology of the metal film is dictated by the quantummechanical states of the electron gas in the metal overlayer. Such states can only occur in the Ag film if there is a confining barrier at the $\mathrm{Ag}$-semiconductor interface. The other confining barrier is obviously the Ag-vacuum interface. The presence of a confining barrier at the Ag-semiconductor interface can be estimated using the so-called Schottky-Mott rule. $^{5,6}$ It states that the barrier height for electron in the metal film to cross the interface into the conduction-band states of the semiconductor is (in a one-dimensional model) the difference of the affinity of the semiconductor $\chi_{s}$ and the work function of the metal $\phi$. The affinity can be determined from the ionization energy $I$ (taken from Refs. 7-11) by subtracting the band gap $E_{G}$ of the semiconductor taken from Ref. 12. At this stage we note that so far the application of the Schottky-Mott rule encountered problems for most metal films on III-V semiconductor. ${ }^{4}$ The reasons for this are that the Schottky-Mott rule assumes that the interface exhibits no interface states in the band gap, such as metal-induced or defect states, and the metal overlayer forms an ideal twodimensional atomically flat film. For most metal coverages of III-V semiconductors, interface states exist, however, and furthermore, the metal films typically do not exhibit a twodimensional but rather a three-dimensional morphology. In view of no detailed model we nevertheless use the SchottkyMott rule in our discussion, because in our case $\mathrm{Ag}$ on the (110) cleavage surfaces of III-V semiconductors forms truely flat Ag films that exhibit thus nearly the morphology of an ideal Ag-semiconductor interface. Furthermore, the predictions of the rule agree qualitatively with the observations and help in the understanding. In the case of $\mathrm{Ag}$ films on GaAs (using $\phi_{\mathrm{Ag}}=4.46 \mathrm{eV}$ ) a confining barrier of $0.33 \mathrm{eV}$ is obtained at the metal-semiconductor interface. This barrier is sufficiently high to confine the electrons in the silver film and thus quantum-mechanical states can develop. We applied this model to all semiconductor substrates used in our study. The affinity of the semiconductor surfaces as well as the barrier heights at the interfaces predicted by the Schottky-Mott rule are shown in Table I. The barrier height estimation using the Schottky-Mott rule shows positive barrier heights for $\mathrm{Ag}$ films on all Ga-containing III-V semiconductors, but negative barrier heights for all In-containing III-V semiconductors. Thus there is only a good confining barrier present at the $\mathrm{Ag}$ interface with the $\mathrm{Ga}-\mathrm{V}$ semiconductors, but no confining barrier for Ag films on In-V semiconductors and consequently the electronic growth mechanism cannot dictate the film morphology in the latter case.

TABLE I. Comparison of the critical thickness and symmetry of the Ag films on different III-V semiconductor substrates with the substrate lattice constants, the substrate affinity, and the barrier at the semiconductor-Ag interface estimated using the Schottky-Mott rule. The values of the affinity at room temperature (RT) were determined using the ionization energy from Refs. 7-11 and the band-gap values (at RT) from Ref. 12.

\begin{tabular}{lcllll}
\hline \hline Material & $\begin{array}{c}\text { Lattice } \\
\text { constant } \\
(\mathrm{nm})\end{array}$ & $\begin{array}{c}\text { Critical } \\
\text { thickness } \\
(\mathrm{nm})\end{array}$ & \multicolumn{1}{c}{ LEED symmetry } & $\begin{array}{c}\text { Affinity } \chi_{s} \\
\text { at RT } \\
(\mathrm{eV})(\text { Ref. 7) }\end{array}$ & $\phi_{\mathrm{Ag}^{-} \chi_{s}(\mathrm{eV})}$ \\
\hline $\mathrm{GaP}$ & 0.545 & 1.7 & Twofold & 3.74 (Ref. 7) & 0.72 \\
$\mathrm{GaAs}$ & 0.565 & 1.5 & Twofold & 4.13 (Ref. 8) & 0.33 \\
$\mathrm{GaSb}$ & 0.610 & 0.6 & $\begin{array}{l}\text { Threefold } \\
\text { (two domains) }\end{array}$ & 4.12 (Ref. 9) & 0.34 \\
$\mathrm{InP}$ & 0.587 & Disordered & Twofold & 4.50 (Ref. 10) & -0.04 \\
$\mathrm{InAs}$ & 0.605 & Disordered & Twofold & 5.02 (Ref. 11) & -0.56 \\
$\mathrm{InSb}$ & 0.648 & Disordered & Weak threefold & 4.70 (Ref. 11) & -0.24 \\
\hline \hline
\end{tabular}


This result explains well the observation of an increased degree of disorder of the Ag films on InP, InAs, and InSb (110) surfaces and of the well-ordered films on Ga-V semiconductors. $\operatorname{InP}(110)$ is as substrate material just at the limit of having a barrier. In fact at the $\mathrm{Ag}$ deposition temperature there is a small positive barrier, but due to the temperature dependence of the band gap it disappears at room temperature. This may be the reason, why the Ag films on InP are still the best ordered ones (with a short range order) of all $\mathrm{Ag}$ films on In-V semiconductors. On the basis of the missing confining barrier it is obvious that the Ag films on InP, InAs, and InSb (110) do not exhibit a critical thickness, whereas the morphology of $\mathrm{Ag}$ films on $\mathrm{GaAs}, \mathrm{GaP}$, and $\mathrm{GaSb}$ is to a large degree influenced by the quantum-mechanical states of the electron gas in the $\mathrm{Ag}$ film. Our experiments thus support the existence of an electronic growth mechanism.

Although the formation and degree of order of the flat $\mathrm{Ag}$ films on the different substrate materials can be well explained on the basis of the electronic growth model, there are additional properties of the thin films connected to the substrate lattice and possibly chemical effects. The observation of a decrease of the critical thickness of the Ag films with increasing lattice constant from $\mathrm{GaP}$ to $\mathrm{GaSb}$ shows that the substrate material affects the electronic properties of the thin $\mathrm{Ag}$ films beyond the simple picture of a confined electron gas. The barrier heights in Table I indicate that GaAs and $\mathrm{GaSb}$ should have rather equal properties concerning the critical thickness. This is not the case, hence there must be additional effects, which have to be considered in the theoretical description of the $\mathrm{Ag}$ film formation. In this context the issue of possible interface states may alter the effective confining barrier at the interface, leading to the observed critical thickness of the Ag films on the different substrates. Furthermore, the presence of a twofold superstructure of the
Ag films on arsenides and phosphides, but a threefold structure of the Ag films on antimonides indicate that chemical effects may play a role in the exact surface reconstruction and structure of the Ag films. The different symmetries cannot be entirely due to simple lattice size effects, because the lattice constants of $\mathrm{GaSb}$ and InAs are very close to each other, but the structures have a different short-range order. This observation indicates that the atomic and bonding structures at the Ag-semiconductor interface also need to be included for a full theoretical understanding.

\section{CONCLUSIONS}

We investigated the substrate effects on the growth of thin $\mathrm{Ag}$ films on (110) cleavage surfaces of III-V compound semiconductors at low temperatures followed by a roomtemperature annealing. We found well ordered $\mathrm{Ag}$ films with well-defined critical thicknesses on Ga-V semiconductors, whereas only disordered Ag films occur on InP, InAs, and $\mathrm{InSb}$ (110) surfaces. This result is explained by the absence of a confining potential for the electrons in the $\mathrm{Ag}$ film grown on In-V semiconductors, due to the semiconductors' electronic structures. On the other hand there is a good confining potential for $\mathrm{Ag}$ films on $\mathrm{Ga}-\mathrm{V}$ semiconductors. The observed film morphologies support the existence of the electronic growth mechanism. In addition we found evidence that the substrate lattice and chemical structure at the interface affect the critical thickness and the superstructure of the Ag films.

\section{ACKNOWLEDGMENTS}

This work was supported by NSF Grant No. DMR9705406, NSF Science and Technology Center Grant No. CHE-8920120, and Texas Advanced Research Program.
${ }^{1}$ A. R. Smith, K.-J. Chao, and C.-K. Shih, Science 273, 226 (1996).

${ }^{2}$ Zhenyu Zhang, Qian Niu, and C.-K. Shih, Phys. Rev. Lett. 80, 5381 (1998).

${ }^{3}$ A. Evans, M. Alonso, R. Cimino, and K. Horn, Phys. Rev. Lett. 70, 3483 (1993).

${ }^{4}$ W. Mönch, Semiconductor Surfaces and Interfaces, Springer Series in Surface Science Vol. 26, edited by G. Ertl, R. Gomer, and D. L. Mills (Springer, Berlin, 1993).

${ }^{5}$ W. Schottky, Phys. Z. 41, 570 (1940).

${ }^{6}$ N. F. Mott, Proc. Cambridge Philos. Soc. 34, 568 (1938).

${ }^{7}$ Average value from J. van Laar, A. Huijser, and T. L. van Rooy, J. Vac. Sci. Technol. 14, 894 (1977); and G. M. Guichar, C. A. Sebenne, and C. Thuault, Surf. Sci. 86, 789 (1979).

${ }^{8}$ Average value from G. W. Gobeli and F. G. Allen, Phys. Rev. 137, A245 (1965); G. M. Guichar, C. A. Sebenne, and G. A. Garry, Phys. Rev. Lett. 37, 1158 (1976); and J. van Laar, A.
Huijser, and T. L. van Rooy, J. Vac. Sci. Technol. 14, 894 (1977).

${ }^{9}$ Average value from G. W. Gobeli and F. G. Allen, Phys. Rev. 137, A245 (1965); G. M. Guichar, C. A. Sebenne, and C. Thuault, in Proceedings of the Seventh International Vacuum Congress and the 3rd International Conference on Solid Surfaces of the International Union for Vaccum Science, Technique and Applications, Vienna, edited by R. Dobrezemsky, F. Rildeneuer, F. P. Viehböck, and A. Breth (F. Berger \& Sohne, Vienna, 1977), p. 623; and J. van Laar, A. Huijser, and T. L. van Rooy, J. Vac. Sci. Technol. 14, 894 (1977).

${ }^{10}$ Average value from T. E. Fischer, Phys. Rev. 142, A1228 (1966); and J. van Laar, A. Huijser, and T. L. van Rooy, J. Vac. Sci. Technol. 14, 894 (1977).

${ }^{11}$ Average value from G. W. Gobeli and F. G. Allen, Phys. Rev. 137, A245 (1965); and J. van Laar, A. Huijser, and T. L. van Rooy, J. Vac. Sci. Technol. 14, 894 (1977).

${ }^{12}$ CRC Handbook of Chemistry and Physics, 79th ed., edited by D. R. Lide (CRC Press, Boca Raton, FL, 1998). 